\title{
Synthesis and Study of Glutamine Acid Containing
}

\section{Citrates}

Iamze Beshkenadze $e^{1,2}$, Nani Zhorzholiani ${ }^{1,2}$, Maia Gogaladze $e^{1,2 *}$, Spartak Urotadze ${ }^{1,2}$, Lia Gogua ${ }^{1,2}$ and Irine Lomtadze ${ }^{1,2}$

1. Petre Melikishvili Institute of Physical and Organic Chemistry, Laboratory of Problems of Agrarian Chemistry, Ivane Javakhishvili Tbilisi State University, Tbilisi 0186, Georgia

2. Tbilisi State Medical University, Tbilisi 0160, Georgia

\begin{abstract}
Terms of synthesis were defined for the creation of new generation chelate fertilizers and for their experimental testing. Glutamine acid and citric acid containing chelate citrates of the general formulae $\mathrm{M} \cdot \mathrm{gl} \cdot \mathrm{HL} \cdot n \mathrm{HO}$ were synthesized, where, $\mathrm{M}=\mathrm{Mn}$, $\mathrm{Zn}, \mathrm{Fe}, \mathrm{Co}, \mathrm{Cu} ; n=0$; 1; 1.5; gl-glutamine acid; HL-citric acid anion. Identity and composition of synthesized compounds were determined by microelemental analysis, melting temperature and X-ray diffraction analysis. X-ray diffraction analysis of the compounds and glutamine acid and citric acid $\left(\mathrm{H}_{4} \mathrm{~L}\right)$ was used to determine their crystalline structure and roentgen-amorphous and iso-structural orders. Their solubility was studied in various solvents.
\end{abstract}

Key words: Fertilizer, chelate compounds, melting, microelemental analysis, X-ray diffraction study.

\section{Introduction}

Humanity entered the new millennium with serious problems of hunger and insufficient nutrition. Therefore one of the most urgent problems today is creation of ecologically safe, farm produce of high quantity and quality indices, which in its turn is the necessary term for stability of human health. One of the main causes that conditions low quantitative and qualitative indices of agricultural products is a deficit of microelements in plants and soil. This is why the decisive role in a matter of solution of this problem is attributed to the provision of a plant as well as soil by optimal quantity and ratio of microelements in chelate form, since it is known that bio-metals fulfill their function in a live body when they are presented in chelate form and when deficiency is compensated by bio-metals in chelate form, biological activity of a live organisms sharply increases [1-7]. Proceeding from

\footnotetext{
*Corresponding author: Maia Gogaladze, Associate Professor, research fields: coordination chemistry, veterinary, agrochemistry and medicine. E-mail: gogaladzemaia@yandex.com.
}

the above stated, significance of fertilizers, which contain organic substances (vitamins, amino acids, oxy-acids) and microelements, for the growth and development of agricultural crops and quantitative and qualitative indices of the product obtained therein is theoretically and practically proved today [8-12].

Irrespective of the above stated, determination of methods of synthesis of chelate compounds which will contain oxy-acids (citric acid), amino acids (glutamine acid) and microelements ( $\mathrm{Zn}, \mathrm{Co}, \mathrm{Fe}, \mathrm{Mn}, \mathrm{Cu}$ ), investigation of physical and chemical properties of the synthesized compounds and determination of efficiency of their application as fertilizers is still actual.

\section{Computational Methods}

To obtain chelate compounds we have used the methods developed, refined and simplified by us. Composition of compounds and their identity was proved by microelement analysis (CHN Analyser, Labertherm $\mathrm{GmbH}$ ). Purity-by defining the melting point (Dynalon SMP10) and by X-ray diffraction 
method, by DPOH-3M type diffractometer, by copper anode emission. The same method was used to determine their crystalline structure, roentgen-amorphous and iso-structural orders were revealed. Qualitative solubility of the synthesized compounds have been studied in various solvents.

\section{Results and Discussion}

Investigations are in progress in the sphere of creation of the new generation fertilizers and their experimental testing at the Laboratory of Problems of Agrarian Chemistry [13-20]. Thus, we synthesized glutamine acid-containing chelate citrates which had the following general formula: $\mathrm{M} \cdot \mathrm{gl} \cdot \mathrm{HL} \cdot n \mathrm{H}_{2} \mathrm{O}$, where, $\mathrm{M}=\mathrm{Mn}$ (1), Zn (2), Fe (3), Co (4), Cu (5); $n=0 ; 1$; 1.5; gl = glutamine acid; HL-citric acid anion. We take $\mathrm{MCO}_{3} \mathrm{M}(\mathrm{OH})_{2}: \mathrm{HL}$ mix in molar ratio $1: 2$ on a porcelain cup to receive (1), (4) and (5) compounds and take $\mathrm{ZnO}: \mathrm{HL}$ and $\mathrm{Fe}\left(\mathrm{CH}_{3} \mathrm{COO}\right)_{2} \cdot 4 \mathrm{H}_{2} \mathrm{O}: \mathrm{HL}$ by $1: 1$ molar ratio to receive (2) and (3), correspondingly, and triturate them separately and mix intensely till homogeneous mass is obtained, then add small quantity of hot water at the terms of intense mixing. In case of (1), (4) and (5), we observe release of $\mathrm{CO}_{2}$, while in case of (3) we observe acute release of acetic acid. After treatment by water several times we add glutamine acid to (1), (4) and (5) at 1:2 molar ratio, then heat each of them, mix and stand on water vapor bath till complete drying of the compound (treat it several times by hot water), wash by ether and dry at room temperature.

Identity and composition of the synthesized chelate compounds $\mathrm{Mn} \cdot \mathrm{gl} \cdot \mathrm{HL}$, Zn·gl $\cdot \mathrm{HL}$, Fe.gl $\cdot \mathrm{HL} \cdot 1.5 \mathrm{H}_{2} \mathrm{O}$, $\mathrm{Co} \cdot \mathrm{gl} \cdot \mathrm{HL} \cdot 1.5 \mathrm{H}_{2} \mathrm{O}, \mathrm{Cu} \cdot \mathrm{gl} \cdot \mathrm{HL} \cdot \mathrm{H}_{2} \mathrm{O}$ were determined by microelement analysis (Table 1 ), by measuring of melting point (Table 2) and X-ray diffraction analysis (Table 3). By X-ray diffraction study of the compounds and its constituents, bioorganic ligands, glutamine acid (gl) and citric acid $\left(\mathrm{H}_{4} \mathrm{~L}\right)$, their crystalline structure and roentgen-amorphous and iso-structural orders were determined.

Table 3 offers the results of relative intensities $\left(I / I_{o}\right)$, reflection angle $(2 \theta)$, inter-planar spacing $(d)$ obtained by deciphering of diffraction patterns of compounds Continuation.

Table 4 offers the results of measuring qualitative solubility of chelate compounds in various solvents.

\section{Discussions}

Microelemental analysis and melting temperature determination proved composition and identity of synthesized compounds.

Table 1 Results of microelemental analysis of chelate compounds.

\begin{tabular}{|c|c|c|c|c|c|c|c|c|c|}
\hline \multirow{2}{*}{ \# } & \multirow{2}{*}{ Formula } & \multicolumn{4}{|c|}{ Practical (\%) } & \multicolumn{4}{|c|}{ Theoretical (\%) } \\
\hline & & M & $\mathrm{C}$ & $\mathrm{H}$ & $\mathrm{N}$ & M & $\mathrm{C}$ & $\mathrm{H}$ & $\mathrm{N}$ \\
\hline 1 & $\mathrm{Mn} \cdot \mathrm{gl} \cdot \mathrm{HL}$ & 14.01 & 33.25 & 4.00 & 3.07 & 13.95 & 33.48 & 3.8 & 3.55 \\
\hline 2 & $\mathrm{Zn} \cdot \mathrm{gl} \cdot \mathrm{HL}$ & 16.06 & 32.14 & 3.15 & 3.88 & 16.08 & 32.65 & 3.71 & 3.46 \\
\hline 3 & $\mathrm{Fe} \cdot \mathrm{gl} \cdot \mathrm{HL} \cdot 1.5 \mathrm{H}_{2} \mathrm{O}$ & 14.99 & 33.75 & 4.09 & 3.26 & 14.17 & 33.39 & 4.55 & 3.54 \\
\hline 4 & $\mathrm{Co} \cdot \mathrm{gl} \cdot \mathrm{HL} \cdot 1.5 \mathrm{H}_{2} \mathrm{O}$ & 13.57 & 30.77 & 3.95 & 3.57 & 13.85 & 31.04 & 4.23 & 3.29 \\
\hline 5 & $\mathrm{Cu} \cdot \mathrm{gl} \cdot \mathrm{HL} \cdot \mathrm{H}_{2} \mathrm{O}$ & 14.76 & 30.02 & 3.86 & 3.07 & 15.16 & 31.52 & 4.06 & 3.34 \\
\hline
\end{tabular}

Table 2 Results of measuring of melting temperatures of chelate compounds.

\begin{tabular}{llll}
\hline$\#$ & Formula & Melting $T\left({ }^{\circ} \mathrm{C}\right)$ & Colour \\
\hline 1 & $\mathrm{Mn} \cdot \mathrm{gl} \cdot \mathrm{HL}$ & 200 & Light cream \\
2 & $\mathrm{Zn} \cdot \mathrm{gl} \cdot \mathrm{HL}$ & 203 & White \\
3 & $\mathrm{Fe} \cdot \mathrm{gl} \cdot \mathrm{HL} \cdot 1.5 \mathrm{H}_{2} \mathrm{O}$ & 210 & Light brown \\
4 & $\mathrm{Co} \cdot \mathrm{gl} \cdot \mathrm{HL} \cdot 1.5 \mathrm{H}_{2} \mathrm{O}$ & 199 & Pink \\
5 & $\mathrm{Cu} \cdot \mathrm{gl} \cdot \mathrm{HL} \cdot \mathrm{H}_{2} \mathrm{O}$ & $>286$ & Dark blue \\
\hline
\end{tabular}


Table 3 Results of X-ray diffraction studies of chelate compounds.

\begin{tabular}{|c|c|c|c|c|c|c|c|c|c|c|c|}
\hline \multicolumn{3}{|c|}{$\mathrm{Mn} \cdot \mathrm{gl} \cdot \mathrm{HL}$} & \multicolumn{3}{|c|}{$\mathrm{Zn} \cdot \mathrm{gl} \cdot \mathrm{HL}$} & \multicolumn{3}{|c|}{$\mathrm{Cu} \cdot \mathrm{gl} \cdot \mathrm{HL} \cdot \mathrm{H}_{2} \mathrm{O}$} & \multicolumn{3}{|c|}{$\mathrm{Co} \cdot \mathrm{gl} \cdot \mathrm{HL} \cdot 1.5 \mathrm{H}_{2} \mathrm{O}$} \\
\hline $2 \theta$ & $D$ & $I / I_{0}$ & $2 \theta$ & $D$ & $J / J_{0}$ & $2 \theta$ & $d$ & $J / J_{0}$ & $2 \theta$ & $d$ & $J / J_{0}$ \\
\hline 5.13 & 17.218 & 1.0892 & 5.19 & 17.027 & 0.7767 & 10.85 & 8.154 & 0.2144 & 10.80 & 8.192 & 0.3615 \\
\hline 10.79 & 8.201 & 0,7321 & 7.74 & 11.422 & 0.9328 & 12.31 & 7.190 & 0.1566 & 11.01 & 8.036 & 0.8384 \\
\hline 13.16 & 6.729 & 0.3714 & 9.03 & 9.793 & 0.3357 & 13.33 & 6.642 & 0.0955 & 13.22 & 6.697 & 0.4076 \\
\hline 15.68 & 5.651 & 1 & 10.34 & 8.555 & 0.3728 & 15.80 & 5.609 & 1 & 13.40 & 6.607 & 0.700 \\
\hline 16.22 & 5.465 & 0.1357 & 12.26 & 7.219 & 0.5271 & 17.11 & 5.182 & 0.1366 & 15.90 & 5.574 & 0.9384 \\
\hline 17.10 & 5.185 & 0.2883 & 15.53 & 5.706 & 0.1614 & 18.35 & 4.835 & 0.1000 & 17.25 & 5.140 & 0.3923 \\
\hline 18,29 & 4.849 & 0.3714 & 17.29 & 5.129 & 0.1557 & 19.35 & 4.587 & 0.0577 & 18.56 & 4.780 & 0.3923 \\
\hline 19.82 & 4.480 & 0.1214 & 18.09 & 4.904 & 0.3642 & 19.82 & 4.479 & 0.1800 & 20.19 & 4.398 & 0.2846 \\
\hline 20.21 & 4.393 & 0.0428 & 20.07 & 4.424 & 1 & 21.80 & 4.077 & 0.1211 & 20.77 & 4.277 & 0.5538 \\
\hline 20.68 & 4.295 & 0.1642 & 20.34 & 4.366 & 0.3900 & 23.15 & 3.842 & 0.1422 & 21.83 & 4.071 & 0.6076 \\
\hline 21.58 & 4.118 & 0.8428 & 20.74 & 4.283 & 0.6671 & 23.84 & 3.732 & 0.0377 & 22.02 & 4.036 & 0.3769 \\
\hline 22.24 & 3.998 & 0.1750 & 21.60 & 4.114 & 0.3328 & 24.62 & 3.616 & 0.0566 & 23.05 & 3.858 & 0.1615 \\
\hline 22.67 & 3.922 & 0.1000 & 22.00 & 4.040 & 0.0928 & 25.46 & 3.498 & 0.0977 & 23.92 & 3.720 & 0.400 \\
\hline 23.60 & 3.769 & 0.3178 & 22.22 & 4.001 & 0.2114 & 26.41 & 3.375 & 0.1944 & 26.51 & 3.362 & 1 \\
\hline 24.02 & 3.705 & 0.0571 & 23.34 & 3.811 & 0.0800 & 29.03 & 3.076 & 0.0722 & 29.15 & 3.063 & 0.2769 \\
\hline 26.04 & 3.421 & 0.1321 & 24.43 & 3.643 & 0.3200 & 30.52 & 2.929 & 0.0600 & 30.23 & 2.956 & 0.2846 \\
\hline 26.23 & 3.397 & 0.8464 & 25.86 & 3.445 & 0.1357 & 31.05 & 2.880 & 0.0933 & 31.11 & 2.875 & 0.9461 \\
\hline 29.07 & 3.072 & 0.1142 & 26.38 & 3.378 & 0.0857 & 31.85 & 2.810 & 0.0788 & 32.02 & 2.795 & 0.1307 \\
\hline 30.54 & 2.927 & 0.5714 & 26.84 & 3.322 & 0.0714 & 34.04 & 2.634 & 0.0854 & 33.92 & 2.643 & 0.2384 \\
\hline 31.25 & 2.862 & 0.1964 & 27.59 & 3.233 & 0.0500 & 37.35 & 2.408 & 0.0755 & 34.42 & 2.605 & 0.1230 \\
\hline 31.63 & 2.829 & 0.1607 & 28.47 & 3.135 & 0.0242 & 37.82 & 2.379 & 0.0688 & 35.37 & 2.538 & 0.1538 \\
\hline 32.67 & 2.741 & 0.0750 & 28.84 & 3.096 & 0.2014 & 40.47 & 2.229 & 0.0711 & 35.82 & 2.507 & 0.4230 \\
\hline 33.29 & 2.712 & 0.1464 & 29.88 & 2.990 & 0.0842 & 44.29 & 2.045 & 0.0488 & 37.74 & 2.384 & 0.3000 \\
\hline 33.84 & 2.649 & 0.3571 & 30.05 & 2.974 & 0.1442 & 45.80 & 1.981 & 0.0233 & 37.88 & 2.375 & 0.3538 \\
\hline 35.09 & 2.557 & 0.2357 & 30.17 & 2.962 & 0.0714 & 46.96 & 1.935 & 0.0488 & 40.33 & 2.228 & 0.3307 \\
\hline 35.90 & 2.501 & 0.2250 & 30.55 & 2.926 & 0.0371 & 48.72 & 1.869 & 0.0944 & 40.71 & 2.216 & 0.3692 \\
\hline 37.08 & 2.425 & 0.2034 & 31.40 & 2.849 & 0.2785 & 50.20 & 1.817 & 0.0366 & 44.26 & 2.046 & 0.4538 \\
\hline 37.47 & 2.400 & 0.4301 & 32.48 & 2.757 & 0.3857 & & & & 44.57 & 2.033 & 0.3538 \\
\hline 38.25 & 2.353 & 0.3464 & 33.88 & 2.646 & 0.2585 & & & & 47.02 & 1.932 & 0.2153 \\
\hline 40.10 & 2.248 & 0.3254 & 34.91 & 2.570 & 0.0728 & & & & 46.99 & 1.934 & 0.2230 \\
\hline 40.15 & 2.246 & 0.1324 & 35.91 & 2.501 & 0.2385 & & & & 48.71 & 1.869 & 0.2076 \\
\hline 42.39 & 2.132 & 0.0857 & 38.64 & 2.330 & 0.1100 & & & & 48.87 & 1.864 & 0.2528 \\
\hline 42.92 & 2.107 & 0.0392 & 38.91 & 2.315 & 0.0714 & & & & 54.69 & 1.678 & 0.0923 \\
\hline 43.86 & 2.064 & 0.2964 & 39.81 & 2.264 & 0.0814 & & & & & & \\
\hline 44.69 & 2.028 & 0.0678 & 40.64 & 2.220 & 0.1000 & & & & & & \\
\hline 46.62 & 1.948 & 0.1821 & 41.22 & 2.190 & 0.1442 & & & & & & \\
\hline 48.16 & 1.889 & 0.1392 & 43.16 & 2.096 & 0.1327 & & & & & & \\
\hline 48.61 & 1.873 & 0.0392 & 44.74 & 2.026 & 0.1800 & & & & & & \\
\hline 49.25 & 1.850 & 0.1142 & 46.34 & 1.959 & 0.0900 & & & & & & \\
\hline 50.57 & 1.805 & 0.0857 & 47.57 & 1.911 & 0.0600 & & & & & & \\
\hline 52.68 & 1.738 & 0.1107 & 48.26 & 1.886 & 0.0447 & & & & & & \\
\hline 53.07 & 1.704 & 0.1142 & 49.34 & 1.847 & 0.0300 & & & & & & \\
\hline 54.58 & 1.681 & 0.0642 & 50.34 & 1.813 & 0.0514 & & & & & & \\
\hline 57.92 & 1.592 & 0.0285 & & & & & & & & & \\
\hline
\end{tabular}




\begin{tabular}{llllll}
\hline & \multicolumn{2}{c}{$\mathrm{H}_{4} \mathrm{~L}$} & & \multicolumn{3}{c}{$\mathrm{gl}$} \\
\hline $2 \theta$ & $d$ & $J / J_{0}$ & $2 \theta$ & $d$ & $J / J_{0}$ \\
\hline 11.5 & 0.3 & 2.5 & 10.2 & 8.672 & 12.0 \\
15.2 & 4.5 & 37.5 & 18.2 & 4.874 & 82.7 \\
17 & 1.6 & 13.3 & 20.4 & 4.353 & 30.7 \\
18.3 & 6.5 & 54.2 & 21.5 & 4.133 & 10.7 \\
19 & 0.4 & 3.3 & 22 & 4.040 & 24.0 \\
22.3 & 4.5 & 37.5 & 23.8 & 3.738 & 100.0 \\
23.1 & 12 & 100.0 & 26.7 & 3.339 & 18.7 \\
23.8 & 0.5 & 4.2 & 28.6 & 3.121 & 4.0 \\
24.5 & 1 & 8.3 & 30 & 2.978 & 8.0 \\
27.1 & 0.5 & 4.2 & 31 & 2.885 & 6.7 \\
28.8 & 0.4 & 3.3 & 32.5 & 2.755 & 33.3 \\
29.4 & 2.5 & 20.8 & 33.2 & 2.698 & 25.3 \\
30.8 & 1.5 & 12.5 & 33.8 & 2.652 & 8.0 \\
32.7 & 1.5 & 12.5 & 35.6 & 2.522 & 10.7 \\
33.5 & 0.8 & 6.7 & 37.1 & 2.423 & 34.7 \\
37 & 1 & 8.3 & 38.8 & 2.321 & 5.3 \\
41.9 & 0.5 & 4.2 & 40.6 & 2.222 & 2.014 \\
45.8 & 0.5 & 4.2 & 45 & 1.873 & \\
56.8 & 0.5 & 4.2 & 48.6 & & \\
\hline
\end{tabular}

Table 4 Qualitative solubility of chelate compounds.

\begin{tabular}{|c|c|c|c|c|c|}
\hline \multirow{2}{*}{ \# } & \multirow{2}{*}{ Compound } & \multicolumn{3}{|c|}{ Solubility } & \multirow{2}{*}{ - Dimethyl formamide } \\
\hline & & Water & Alcohol & Acetone & \\
\hline 1 & $\mathrm{Mn} \cdot \mathrm{gl} \cdot \mathrm{HL}$ & $+\mathrm{t}$ & - & Weakly soluble & - \\
\hline 2 & $\mathrm{Zn} \cdot \mathrm{gl} \cdot \mathrm{HL}$ & $+\mathrm{t}$ & - & - & Weakly soluble \\
\hline 3 & $\mathrm{Fe} \cdot \mathrm{gl} \cdot \mathrm{HL} \cdot 1.5 \mathrm{H}_{2} \mathrm{O}$ & - & - & - & - \\
\hline 4 & $\mathrm{Co} \cdot \mathrm{gl} \cdot \mathrm{HL} \cdot 1.5 \mathrm{H}_{2} \mathrm{O}$ & $+\mathrm{t}$ & Weakly soluble & Weakly soluble & Weakly soluble \\
\hline 5 & $\mathrm{Cu} \cdot \mathrm{gl} \cdot \mathrm{HL} \cdot \mathrm{H}_{2} \mathrm{O}$ & $+\mathrm{t}$ & Weakly soluble & Weakly soluble & Weakly soluble \\
\hline
\end{tabular}

$\mathrm{X}$-ray diffraction investigations showed that presence of starting compounds in the samples is not expressed in the form of separate phase, which enables us to conclude that absolutely new individual compounds are obtained, which is in full conformity with the results of the above stated microelemental analysis and melting temperature measurements (Tables 1 and 2). Roentgenogram of the chelate compound Fe.gl·HL·1.5HO is characterized by weakly expressed diffuse peaks, that the main phase is roentgen-amorphous, on the base of which we can conclude that the above referred compound is roentgen amorphous compound. Other compounds are characterized by clearly expressed crystalline structure, various relative intensities $\left(I / I_{0}\right)$, definite

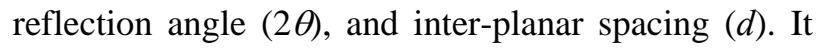

should be stated that roentgenograms of the compounds $\mathrm{Mn} \cdot \mathrm{gl} \cdot \mathrm{HL}, \mathrm{Cu} \cdot \mathrm{gl} \cdot \mathrm{HL} \cdot \mathrm{H}_{2} \mathrm{O}$ are identical, which refers to their iso-structuring and is conditioned by analogousness of their chemical composition and geometrical characteristics of their crystalline structures, which can't be said about the compounds $\mathrm{Zn} \cdot \mathrm{gl} \cdot \mathrm{HL}$, Co.gl·HL·1.5HO (Table 3).

Measurements of qualitative solubility of chelate compounds in various solvents (Table 4) proved that the compounds are dissolved at heating in water, while in organic solvents (alcohol, acetone, dimethyl formamide) are insoluble or are characterized by weak solubility.

\section{Conclusions}

Glutamine acid containing chelate citrates of 
general formula: $\mathrm{M} \cdot \mathrm{gl} \cdot \mathrm{HL} \cdot n \mathrm{HO}$, where, $\mathrm{M}=\mathrm{Mn}(1)$, $\mathrm{Zn}$ (2), Fe (3), Co (4), Cu (5); n = 0-1.5; gl-glutamine acid; HL-citric acid anion were synthesized by the methods developed and simplified by us, in order to create on their base the new generation chelate fertilizers for agricultural crops and to test experimentally. Identity and composition of synthesized compounds were determined. Their crystalline structure was studied, roentgen amorphous iso-structural orders were revealed. Qualitative solubility of the synthesized compounds was determined in various solvents. Fertilizers made on the base of synthesized compounds were tested for maize crop in field conditions. On the base of the obtained results, the conclusion was made about purposefulness to carry out more wide-scale experiments in this direction.

\section{Acknowledgments}

We thank Science \& Technology Center in Ukraine and Shota Rustaveli National Science Foundation. The work was implemented with the support of Science \& Technology Center in Ukraine Project Proposal \#5461 and Shota Rustaveli National Science Foundation Grant \#30/06.

\section{References}

[1] Dobrinina, N. A. 2007. Bioinorganic Chemistry. Lomonosov Moscow State University: Moscow.

[2] Williams, R. J. P. 1999. "Bio-inorganic Chemistry-Its Conceptual Evolution.” Coord. Chem. Rev. 100: 573-610.

[3] Atkins, P., Overton, T., Rourke, J., Weller, M., and Armstrong, F., eds. 2006. Shriver \& Atkins. University Press: Oxford.

[4] Kiselev, Y. M., and Dobrynina, N. A. 2007. Chemistry of Coordination Compounds.

[5] Rybkina, T. I. 1999. "Biological and Toxic Impact of Chemical Elements and Their Inorganic Compounds on Human Body.” Novomoskovsk 92.

[6] Chemical Elements in Human Body http://alhimik.ru/kunst/man's_elem.html

[7] Ershov, Y. A. M., ed. 1998-2000. "Chemistry of Biogenic
Elements.” In General Chemistry.

[8] Microelements-Containing Chelates http://www. sadogorod-na.ru/osnovzemledelija/mineralnie-udobrenija. html\#top

[9] Role of Microelements to Keep in Balance Mineral Nutrition of Agricultural Crops http://www.bhz.kosnet. ru/Rus/Stat/St_2010_03_Chenonogov.html.

[10] Bulygin-Dnepropetrovsk, S. Y., ed.; 2003; Microelements in Agriculture.

[11] Karapetyan, F. O., and Karapetyan, K. G. 2000. "Mineral Fertilizers of XXI Century in the Specter of Ecology Problems." Scientific Technical Records of "SPBGTU" 1: 19.

[12] Organo-Mineral Fertilizer “Universal” www.bhz.Kosnet. ru.

[13] Beshkenadze. Microelement-Containing Chelate Fertilizers. Sak Patent P5, 258, issued Sep. 09, 2011.

[14] Beshkenadze. Chelate Phytoregulator. Sak Patent U1, 746, issued Dec. 14, 2012.

[15] Urotadze, S., Beshkenadze, I., Zhorzholiani, N., Sakvarelidze, T., Gogaladze, M., Barisashvili, M., et al. 2009. "The Phytoregulator Contents of Succinic Acid and Microelements.” Bulletin of the Academy of Agricultural Sciences of Georgia 25: 17-20.

[16] Urotadze, S., Beshkenadze, I., Zhorzholiani, N., Gogaladze, M., and Gogua, L. 2010. Microelements Containing Coordination Compounds and the Phytoregulators Created on their Basis.

[17] Gogaladze, M., Urotadze, S., Beshkenadze, I., Zhorzholiani, N., and Sakvarelidze, T. 2009. "The Phytoregulator Content of Microelements.” In Proceedings of Republican Scientific-Practical Conference Modern Achievements of Applied Chemistry and Technology, 80-82.

[18] Gogaladze, M., Urotadze, S., Beshkenadze, I., Zhorzholiani, N., Barisashvili, M., and Tsereteli, B. 2010. "Modern Fertilizers Containing Microelements." In Proceedings of International Scientific-Practical Conference.

[19] Beshkenadze, I., Urotadze, S., Zhorzholiani, N., and Gogaladze, M. 2011. "A New Generation of Environmentally Safe Phyto Regulators.” In Proceedings of International Scientific-Practical Conference.

[20] Beshkenadze, I., Gogaladze, M., Zhorzholiani, N., Urotadze, S., and Lomtadze, I. 2013. "Study of Microelements-Containing Phyto-Regulator.” In Proceedings of II International Scientific-Practical Conference, Bioeconomy and Sustainable Development of Agriculture. 